\title{
Exome and copy number variation analyses of Mayer-Rokitansky-Küster- Hauser syndrome
}

\author{
Kazumi Takahashi ${ }^{1,2}$, Takahide Hayano ${ }^{3}$, Ryota Sugimoto ${ }^{4}$, Hirofumi Kashiwagi ${ }^{1}$, Mari Shinoda ${ }^{1,2}$, Yoshihiro Nishijima ${ }^{1}$, \\ Takahiro Suzuki ${ }^{1}$, Shingo Suzuki ${ }^{5}$, Yuko Ohnuki ${ }^{2,5}$, Akane Kondo ${ }^{1,2,6}$, Takashi Shiina ${ }^{5}$, Hirofumi Nakaoka ${ }^{4}$, \\ Ituro Inoue ${ }^{4}$ and Shun-ichiro Izumi ${ }^{1,2}$
}

\begin{abstract}
Mayer-Rokitansky-Küster-Hauser (MRKH) syndrome is characterized by congenital absence of the vagina and uterus. We conducted genome-wide SNP analyses and exome sequencing to detect the causes of MRKH syndrome. We identified de novo variants of MYCBP2, NAV3, and PTPN3 in three families and a variant of MYCBP2 in a sporadic case. Here, we demonstrated the partial genetic makeup of Japanese MRKH syndrome.
\end{abstract}

Mayer-Rokitansky-Küster-Hauser (MRKH) syndrome is characterized by the congenital absence of the upper two-thirds of the vagina and uterus and occurring at a rate of 1 in 4500 newborn girls. Patients with MRKH syndrome have a normal female karyotype (46XX) and seemingly normal development of secondary sex characteristics and typically present with amenorrhea during adolescence, resulting in problems with sexual intercourse and infertility ${ }^{1}$. This syndrome is classified into type I and type II: type I involves only uterovaginal aplasia, and type II involves uterovaginal aplasia with concomitant defects, such as renal malformations, skeletal malformations, hearing defects, and rare cardiac and digital anomalies ${ }^{1}$. The etiology of this syndrome remains elusive because most of the cases are sporadic with potential underlying heterogenous causes. However, familial aggregation is occasionally observed, and genetic involvement has been reported by several investigators, showing likely autosomal dominant

Correspondence: Kazumi Takahashi (ellekazu@tokai-u.jp)

'Department of Obstetrics and Gynecology, Tokai University School of Medicine, Isehara, Kanagawa, Japan

${ }^{2}$ Department of Clinical Genetics, Tokai University Hospital, Isehara, Kanagawa, Japan

Full list of author information is available at the end of the article. inheritance with incomplete penetrance ${ }^{1,2}$. Frequent genomic rearrangements, such as deletions and duplications, have been identified by array comparative genome hybridization (CGH) analyses; however, a consistent pattern has not been observed, and a responsive gene has not been pinpointed, except for $L H X 1$ and $H N F 1 B$ on chromosome $17 \mathrm{q} 12^{1,3-5}$. Because large families that promote linkage and positional cloning are mostly lacking in MRKH syndrome, a candidate gene approach has been utilized for detecting causal genes; thus far, only WNT4 has been identified in MRKH syndrome with hyperandrogenism ${ }^{1}$.

Recent advances in sequence technologies have propelled genetic analyses of both rare and common diseases at the whole-genome level. We performed genomewide single-nucleotide polymorphism (SNP) analyses to detect chromosomal rearrangement and exome analysis to identify causal variants in trio-families and sporadic cases.

Informed consent was obtained in accordance with the Declaration of Helsinki, and the study protocol was approved by the Institutional Review Board of Tokai University School of Medicine (12I-03, 17I-32), National Institute of Genetics (nig1608) and Yamaguchi University Graduate School of Medicine (H29-229). Each participant 
gave written informed consent for the collection of samples and subsequent analyses. A total of 17 specimens (ten patients and seven unaffected individuals from the families) were recruited in this study. Six of the ten patients had type I MRKH syndrome, and four of the ten patients had type II MRKH syndrome (Table S1). We also analyzed samples from healthy parents (mother and father) of two patients (A5 and A7) and samples from healthy family members (mother, father, and sister) of one patient (A6). We designated families of A5, A6, and A7 as Fam01, Fam02, and Fam03, respectively (Figure S1).

Genomic DNA was extracted from the peripheral white blood cells of individuals using the QIAamp DNA Mini Kit (Qiagen) according to the manufacturer's instructions. Genomic DNA was hybridized using the SureSelect Human All Exon V5 Kit (Agilent) and sequenced using a HiSeq 2500 (Illumina) with 100 or 150 base paired end modules. Sequencing data were mapped to a human genome reference (hg19) using a standard method of BWA, Picard, and GATK, as previously described ${ }^{6,7}$. Variant calling and genotyping were performed using
GATK HaplotypeCaller ${ }^{6}$. De novo variants in the three families (Fam01, Fam02, and Fam03) were called using the Trio Calling module of VarScan ${ }^{8}$. Allele frequency and functional information of variants were annotated by ANNOVAR ${ }^{9}$. We also used allele frequencies in the Japanese population for further selection of variants using the Human Genetic Variation Database (HGVD) ${ }^{10}$. After selection of variants, the allele frequencies were manually reviewed in the Integrative Japanese Genome Variation Database (iJGVD) $)^{11}$. Single-nucleotide polymorphism (SNP) array experiments using Infinium OmniExpress-24 BeadChips (Illumina) were conducted for nine of the 10 patients with MRKH except for A5. A PennCNVParseCNV analysis pipeline ${ }^{12,13}$ was applied to detect structural variations. To identify disease-specific CNV, we used in-house $\mathrm{CNV}$ data from individuals without MRKH. For Fam01 (A5, C8, and C9), the array-CGH experiment was conducted using the Agilent Human Genome CGH Microarray $1 \mathrm{M}$ Kit.

Three common structural variations were identified by the PennCNV-ParseCNV pipeline (Fig. 1). One amplified
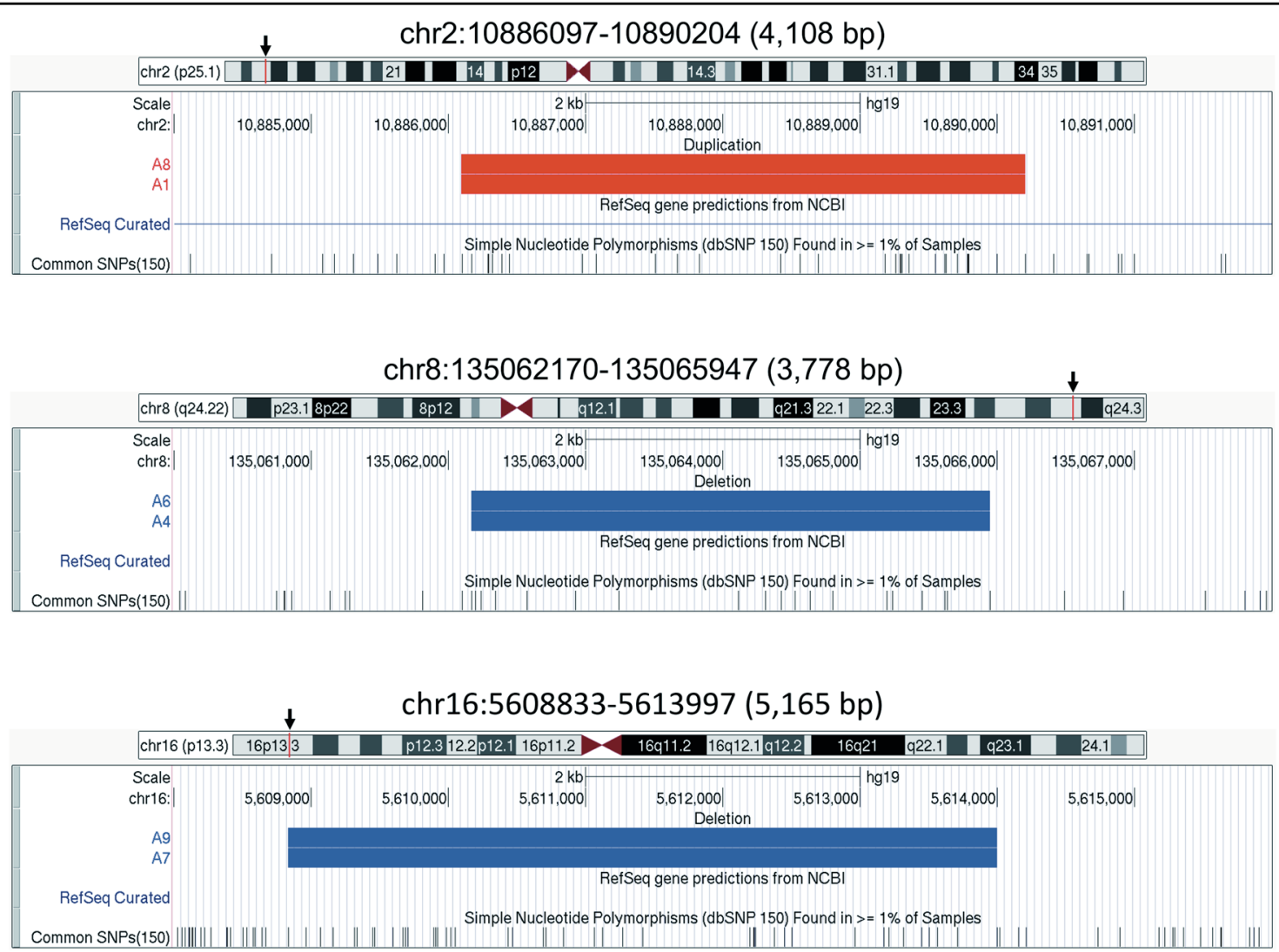

Fig. 1 Three common structural variations of MRKH syndrome. (Top) A duplication (4108 bp) in chromosome 2 (chr2p25.1). A1 and A8 had a common duplication in chr2:10,886,097-10,890,204. (Middle) A deletion (3778 bp) in chromosome 8 (chr8q24.22). A4 and A6 had a common deletion in chr8:135,062,170-135,065,947. (Bottom) A deletion (5165 bp) in chromosome 16 (chr16p13.3). A7 and A9 had a common deletion in chr16:5,608,833-5,613,997. Black arrows and red or blue vertical lines indicate the positions of structural variations in chromosome ideograms. All structural variations were visualized using UCSC Genome Browser (hg19) 
structural variation (chr2:10,886,097-10,890,204) was found in A1 and A8. A4 and A6 shared a deleted region of chr8:135,062,170-135,065,947. A7 and A9 shared a deleted region of chr16:5,608,833-5,613,997. Identified structural variations were not large and were not involved in coding regions. No overlapping region in the reported results and WNT4 region was identified. Because two patients shared the same structural variations at each site, these variations would likely be polymorphic. Therefore, we shifted our focus to singlenucleotide variants (SNVs) and small insertion and deletions (Indels) to evaluate causality using whole-exome analyses mainly in trio-based families.

Ten patients including three patients from trio-based families and seven unaffected individuals from their families were subjected to whole-exome analyses. The mean depth of exome sequencing was $\times 71$ and ranged from $\times 49$ to $\times 98$. Coverage $\geq \times 10$ was achieved for more than $90 \%$ of all the samples (Table S2). A total of 228,135 variants and an average of 95,293 variants (from 91,835 to 97,514) were detected in the 17 samples. After selection of exonic and splicing variants with an allele frequency $\leq 5 \%$, an average of 284 variants (from 247 to 348) remained. Variants were surveyed using the Japanese public database (HGVD), and variants with allelic frequency $<0.1 \%$ were selected (Table S3 and S4). We could not find any variants in the previously reported MRKH-associated genes (LHX1, $H N F 1 B$, and WNT4). To identify de novo variant(s) in the three families, we conducted trio-based de novo variant calling. After manual curation of variants using $\mathrm{IGV}^{14}$, we identified three de novo variants in three genes (MYCBP2, NAV3, and PTPN3) (Table S5). We also identified a distinct variant of $M Y C B P 2$ in a sporadic case (A1). Genotypes of all the de novo variants and the sporadic variant of $M Y C B P 2$ were heterozygous, and the positions of the variants are show$\mathrm{n}$ in Table 1. Functional predictions of variants in

Table 1 Functional predictions of the three de novo variants and MYCBP2 variants in A1

\begin{tabular}{|c|c|c|c|c|}
\hline Paitent & A5 & $\mathrm{A} 1$ & A6 & A7 \\
\hline Position & chr13:77661732 & chr13:77752067 & chr12:78400834 & chr9:112182816 \\
\hline Gene & MYCBP2 & MYCBP2 & NAV3 & PTPN3 \\
\hline NCBI accession & NM_015057 & NM_015057 & NM_001024383 & NM_002829 \\
\hline Protein change & p.T3588A & p.H1719P & p.1506V & p.N401H \\
\hline Genotype (Het/Homo) & Het & Het & Het & Het \\
\hline SIFT_score & 0.101 & 0.053 & 0.068 & 0.893 \\
\hline SIFT_pred & $\mathrm{T}$ & $\mathrm{T}$ & $\mathrm{T}$ & T \\
\hline Polyphen2_HDIV_score & 0.956 & 0.980 & 0.913 & 0.917 \\
\hline Polyphen2_HDIV_pred & $\mathrm{P}$ & D & $P$ & P \\
\hline Polyphen2_HVAR_score & 0.931 & 0.948 & 0.891 & 0.219 \\
\hline Polyphen2_HVAR_pred & D & D & P & B \\
\hline LRT_score & 0.000 & 0.000 & 0.001 & 0.001 \\
\hline LRT_pred & D & D & $U$ & D \\
\hline MutationTaster_score & 1.000 & 1.000 & 1.000 & 0.989 \\
\hline MutationTaster_pred & $\mathrm{D}$ & D & D & D \\
\hline MutationAssessor_score & 1.780 & 0.690 & 2.565 & 1.100 \\
\hline MutationAssessor_pred & $\mathrm{L}$ & N & M & $\mathrm{L}$ \\
\hline FATHMM_score & 1.610 & 1.710 & 1.200 & 1.760 \\
\hline FATHMM_pred & $\mathrm{T}$ & $\mathrm{T}$ & T & $\mathrm{T}$ \\
\hline PROVEAN_score & -2.410 & -3.690 & -0.680 & 0.100 \\
\hline PROVEAN_pred & N & $\mathrm{D}$ & N & N \\
\hline MetaSVM_score & -1.003 & -1.036 & -0.731 & -0.981 \\
\hline MetaSVM_pred & $T$ & $T$ & $\mathrm{~T}$ & T \\
\hline
\end{tabular}


Table 1 continued

\begin{tabular}{lllll}
\hline Paitent & A5 & A1 & A6 & A7 \\
\hline MetaLR_score & 0.121 & 0.100 & 0.218 & 0.130 \\
MetaLR_pred & $T$ & $T$ & $T$ & $T$ \\
M-CAP_score & 0.010 & 0.009 & 0.011 & 0.013 \\
M-CAP_pred & $T$ & $T$ & $T$ & $T$ \\
fathmm-MKL_coding_score & 0.994 & 0.987 & 0.994 & 0.975 \\
fathmm-MKL_coding_pred & $\mathrm{D}$ & $\mathrm{D}$ & $\mathrm{D}$ \\
GERP ++_RS & 5.440 & 5.720 & 5.490 & 4.800 \\
\hline
\end{tabular}

Twelve functional prediction scores (SIFT, Polyphen2_HDIV, Polyphen2_HVAR, LRT, MutationTaster, MutationAssessor, FATHMM, PORVEAN, MetaSVM, MetaLR, M-CAP, and fathmm-MKL) and one evolutionally conservation score (GERPP ++ _RS) were annotated using ANNOVAR

Het Heterozygote, Homo Homozygote, $T$ tolerated, $D$ damaging or deleterious, $P$ probably damaging, $B$ benign, $U$ unknown, $L / N$ non-functional, $M$ medium

MYCBP2 (A1 and A5), NAV3 (A6), and PTPN3 (A7) are summarized in Table 1 . All the identified variants havenot been registered in the most updated databases, and all the variants are probably deleterious (especially predicted by MutationTaster and fathmm-MKL). MYCBP2 is located in chromosome 13q22.3, which has not yet been identified to harbor SNVs and chromosome aberrations in MRKH syndrome. MYCBP2 encodes an E3 ubiquitin-protein ligase and has not been described in the etiology of uterine and vaginal development ${ }^{15}$. Reduced expression of $M Y C B P 2$ has been observed in acute lymphoblastic leukemia patients ${ }^{16}$, and a deletion mutation in this gene has been associated with developmental abnormality of optical discs, resulting in a rare inherited vision defect ${ }^{17}$. NAV3 is located on chromosome 12, and it belongs to a neuron navigator family that is expressed in the nervous system ${ }^{18}$. Because $N A V 3$ variants have not been implicated in diseases and only one patient had the variant, $N A V 3$ was not considered to be a cause of MRKH. PTPN3 is located on chromosome 9, and it belongs to a tyrosine phosphatase family ${ }^{19}$. PTPN3 has multiple functions in cellular process, such as differentiation and growth. Somatic mutation of PTPN3 can promote cell proliferation and cholangiocarcinoma ${ }^{20}$, but the involvement of this gene in MRKH is not clear.

Genetic approaches to identify the genetic causalities for MRKH syndrome have not been successful. Thus, the focus should be on epigenetic and environmental factors underlying the disease. Indeed, discordant phenotype in twin sisters has been reported, indicating more heterogenous characteristics of the syndrome. Therefore, genetic and nongenetic factors need to be investigated for full understanding of MRKH syndrome.

We identified two mutations in MYCBP2 in two patients (A1 and A5). In particular, one patient (A5) showed a de novo mutation. The functional involvement of $M Y C B P 2$ in the etiology of MRKH syndrome needs to be further investigated.

\section{HGV database}

The relevant data from this Data Report are hosted at the Human Genome Variation Database at

https://doi.org/10.6084/m9.figshare.hgv.2378

https://doi.org/10.6084/m9.figshare.hgv.2381

https://doi.org/10.6084/m9.figshare.hgv.2384

https://doi.org/10.6084/m9.figshare.hgv.2387

\section{Acknowledgements}

We would like to thank the patients and their families for participating in this study. We also would like to thank the following individuals for their technical support: Atsuko Sigenari and Sayaka Ito in the Department of Molecular Life Science in Tokai University School of Medicine; Masayuki Tanaka in the Support Center for Medical research and Education in Tokai University School of Medicine; and Maki Moritani in the Department of Clinical Research in Shikoku Medical Center for Children and Adults.

\section{Author details}

${ }^{1}$ Department of Obstetrics and Gynecology, Tokai University School of Medicine, Isehara, Kanagawa, Japan. ${ }^{2}$ Department of Clinical Genetics, Tokai University Hospital, Isehara, Kanagawa, Japan. ${ }^{3}$ Department of Systems Bioinformatics, Yamaguchi University Graduate School of Medicine, Ube, Japan. ${ }^{4}$ Division of Human Genetics, National Institute of Genetics, Mishima, Shizuoka, Japan. ${ }^{5}$ Department of Molecular Life Science, Division of Basic Medical Science and Molecular Medicine, Tokai University School of Medicine, Isehara, Kanagawa, Japan. ${ }^{6}$ Perinatal Medical Center, Shikoku Medical Center for Children and Adults, National Hospital Organization, Zentsuji, Kagawa, Japan

Conflict of interest

The authors declare that they have no conflict of interest.

\section{Publisher's note}

Springer Nature remains neutral with regard to jurisdictional claims in published maps and institutional affiliations.

Supplementary information is available for this paper at https://doi.org/ 10.1038/s41439-018-0028-4.

Received: 2 July 2018 Revised: 18 August 2018 Accepted: 20 August 2018 Published online: 27 September 2018

\section{References}

1. Ledig, S. \& Wieacker, P. Clinical and genetic aspects of Mayer-RokitanskyKüster-Hauser syndrome. Med. Genet. 30, 3-11 (2018).

2. Herlin, M., Højland, A. T. \& Petersen, M. B. Familial occurrence of MayerRokitansky-Küster-Hauser syndrome: a case report and review of the literature. Am. J. Med. Genet. A. 164A, 2276-2286 (2014). 
3. Bernardini, L. et al. Recurrent microdeletion at $17 q 12$ as a cause of MayerRokitansky-Kuster-Hauser (MRKH) syndrome: two case reports. Orphanet J. Rare Dis. 4, 25 (2009)

4. Ledig, S. et al. Recurrent aberrations identified by array-CGH in patients with Mayer-Rokitansky-Küster-Hauser syndrome. Fertil. Steril. 95, 1589-1594 (2011).

5. Demir Eksi, D. et al. Copy number variation and regions of homozygosity analysis in patients with MÜLLERIAN aplasia. Mol. Cytogenet. 11, 13 (2018).

6. Van der Auwera, G. A. et al. From FastQ data to high confidence variant calls: the Genome Analysis Toolkit best practices pipeline. Curr. Protoc. Bioinformatics 43, 11.10.1-33 (2013).

7. Hayano, T. et al. Germline variants of prostate cancer in Japanese families. PLoS ONE 11, e0164233 (2016).

8. Koboldt, D. C., Larson, D. E. \& Wilson, R. K. Using VarScan 2 for germline variant calling and somatic mutation detection. Curr. Protoc. Bioinformatics 44, 15.4.1-17 (2013).

9. Yang, H. \& Wang, K. Genomic variant annotation and prioritization with ANNOVAR and WANNOVAR. Nat. Protoc. 10, 1556-1566 (2015).

10. Higasa, K. et al. Human genetic variation database, a reference database of genetic variations in the Japanese population. J. Hum. Genet. 61, 547-553 (2016).

11. Yamaguchi-Kabata, Y. et al. iJGVD: an integrative Japanese genome variation database based on whole-genome sequencing. Hum. Genome Var. 2, 15050 (2015).
12. Glessner, J. T., Li, J. \& Hakonarson, H. ParseCNV integrative copy number variation association software with quality tracking. Nucleic Acids Res. 41, e64 (2013).

13. Hayano, T. et al. Molecular characterization of an intact p53 pathway subtype in high-grade serous ovarian cancer. PLOS ONE 9, e114491 (2014).

14. Robinson, J. T., Thorvaldsdóttir, H., Wenger, A. M., Zehir, A. \& Mesirov, J. P. Variant review with the integrative genomics Viewer. Cancer Res. 77, e31-e34 (2017).

15. Pao, K. C. et al. Activity-based E3 ligase profiling uncovers an E3 ligase with esterification activity. Nature 556, 381-385 (2018).

16. Ge, Z. et al. Clinical significance of high C-MYC and low MYCBP2 expression and their association with Ikaros dysfunction in adult acute lymphoblastic leukemia. Oncotarget 6, 42300-42311 (2015).

17. Bredrup, C. et al. High myopia-excavated optic disc anomaly associated with a frameshift mutation in the MYC-binding protein 2 gene (MYCBP2). Am. J. Ophthalmol. 159, 973-979 (2015).

18. Carlsson, E. et al. Neuron navigator 3 alterations in nervous system tumors associate with tumor malignancy grade and prognosis. Genes Chromosomes Cancer 52, 191-201 (2013).

19. Parker, E. J. The molecular basis for the substrate specificity of protein tyrosine phosphatase PTPN3. Structure 23, 608-609 (2015).

20. Gao, Q. et al. Activating mutations in PTPN3 promote cholangiocarcinoma cel proliferation and migration and are associated with tumor recurrence in patients. Gastroenterology 146, 1397-1407 (2014). 